\title{
Equilibration chronometry: characterizing neutron-proton equilibration within a strongly deformed nuclear system with sub-zeptosecond resolution
}

\author{
A. Jedele* \\ Cyclotron Institute, Texas A\&M University \\ Chemistry Department, Texas A\&M University \\ E-mail: ajedele@tamu.edu
}

\section{A.B. Mclntosh}

Cyclotron Institute, Texas A\&M University

Email: alan.b.mcintoshegmail.com

\section{K. Hagel, E. McCleskey, A. Rodriguez Manso and M. Youngs}

Cyclotron Institute, Texas A\&M University

\section{Heilborn, Z. Kohley, L.W. May, A. Zarrella and S.J. Yennello \\ Cyclotron Institute, Texas A\&M University \\ Chemistry Department, Texas A\&M University}

\begin{abstract}
Neutron-proton equilibration was studied in dynamically-deformed nuclei produced in reactions ${ }^{70} \mathrm{Zn}+{ }^{70} \mathrm{Zn}$ at $35 \mathrm{~A} \mathrm{MeV}$. The composition of the two largest daughters of each excited projectilelike fragment is examined as a function of alignment angle. The lighter fragment is initially neutron rich relative to the heavier fragment, and the compositions evolve towards each other exponentially with increasing angle. The alignment angle, as a result of rotation, is related to time through the angular momentum of the projectile-like fragment, and a rate constant of $3 \mathrm{zs}^{-1}$ is extracted. This corresponds to a mean equilibration time of $0.3 \mathrm{zs}$. This technique provides a new window through which to gain insight into the nuclear equation of state.
\end{abstract}

The 26th International Nuclear Physics Conference

11-16 September, 2016

Adelaide, Australia

${ }^{*}$ Speaker. 
The nuclear equation of state $(\mathrm{nEoS})$ relates thermodynamic state variables for nuclear matter. Implications for understanding the $\mathrm{nEoS}$ range from microscopic to astronomical. Microscopic implications include understanding collective excitations of nuclei, neutron skin thicknesses of nuclei and dynamics of heavy-ion collisions $[1,2,3]$. Astronomically, the $\mathrm{nEoS}$ influences the radius and crust composition of neutron stars $[4,5]$. The $n E o S$ for stable nuclei near saturation density has been well constrained.

The symmetry energy is the portion of the $\mathrm{nEoS}$ that describes the penalty for having a relative excess of neutrons or protons. The density dependence of this energy penalty is the least well constrained term in the nEoS. In the laboratory, this can be probed by creating nuclear matter at sub- and supra-saturation density through heavy ion collisions. Several probes have been used to study this density-dependence include neutron-proton ratios [6,7], isoscaling [8, 9, 10], isospin diffusion [11, 12, 13], and neck dynamics and emission [14, 15, 16, 17]. Recently, the timedependence of neutron-proton (NZ) equilibraton has re-emerged as an additional probe.

Experiments initially probing the timescale of NZ equlibration $[18,19,20]$ in the 1970s and 1980 s established a timescale between $0.1-1.0$ zs ( 1 zeptosecond is $10^{-21}$ seconds). These experiments measured the mass composition for nuclei of select atomic number as a function of the energy dissipation or separation angle. The interaction time between the target and projectile is related to the energy dissipation. Recent measurements by Hudan et al. [21, 22, 23] used the separation angle as a surrogate for the interaction time, which can be calculated using the angular momentum. Results showed signs of equilibration persisting up to $4 \mathrm{zs}$, although most of the equilibration occurs within $1 \mathrm{zs}$.

In this proceeding, evidence of neutron-proton equilibration between nuclei on a sub-zeptosecond timescale is presented. The equilibration chronometry technique, pioneered by Hudan et al., was applied to experimental data with almost complete angular coverage and excellent isotopic resolution. The evolution of the NZ equilibration was observed in $0.1 \mathrm{zs}$ time slices.

$\mathrm{A}{ }^{70} \mathrm{Zn}$ beam was accelerated to $35 \mathrm{~A} \mathrm{MeV}$ by the $\mathrm{K} 500$ at the Cyclotron Institute at Texas A\&M University. The beam was impinged on a ${ }^{70} \mathrm{Zn}$ thin foil target. Charged products of the nuclear reaction were measured in NIMROD (Neutron Ion Multidetector for Reaction Oriented Dynamics) [24]. NIMROD has nearly $4 \pi$ coverage from $3.6^{\circ}-167.0^{\circ}$. The detector array has excellent isotopic resolution up to $\mathrm{Z}=17$ for most detectors and $\mathrm{Z}=21$ for select high-performing detectors. Elemental identification is achieved through the atomic number of the beam. The combination of the isotopic identification and almost complete geometric coverage allows for coincident measurement of the composition of the two heaviest fragments of the excited projectile-like fragment $\left(\mathrm{PLF}^{*}\right)$ with high resolution.

In the energy regime of $35 \mathrm{~A} \mathrm{MeV}$, nuclear reactions primarily result in the formation of an excited projectile-like fragment ( $\left.\mathrm{PLF}^{*}\right)$ and an excited target-like fragment (TLF*). After colliding, and as the two fragments begin to separate, a neck region of nuclear matter forms as depicted in Figure 1a. Due to collision dynamics, the system becomes stretched, and attractive forces can no longer hold the system together causing it to break apart. The resulting PLF* and TLF* are strongly deformed along their axis of separation, and as a result, are likely to break-up again. The deformed PLF* shortly before breaking up is shown in Figure 1b. break-ups resulting from the strong deformation of the PLF* or TLF* are referred to as dynamical decay and have a characteristic angular distribution aligned close to the beam direction $[17,14,15]$. This analysis focuses 


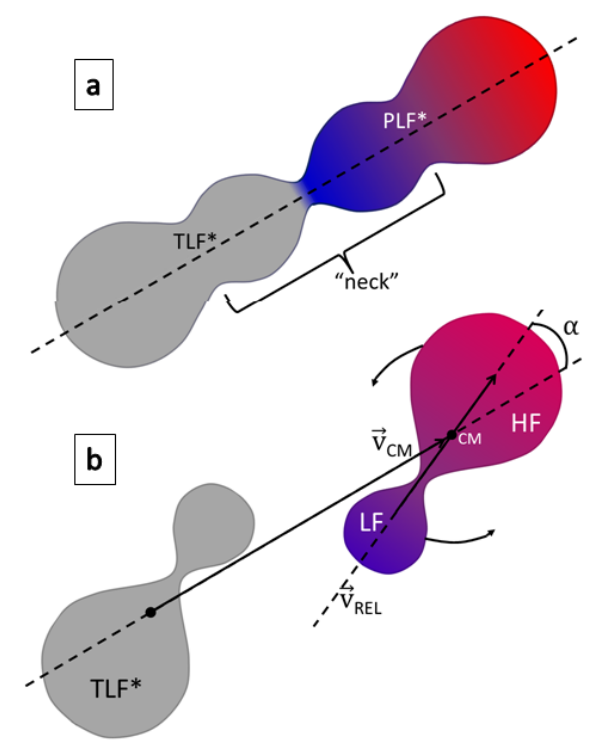

Figure 1: Depiction of the origin of dynamical deformation and its decay. Panel a shows the deformed PLF* and TLF* system before rupturing. Panel b shows a latter time where the PLF* and TLF* have broken apart. The PLF* has rotated relative to $\overrightarrow{v_{C M}}$. The PLF* is about to break apart into twp fragments (HF and LF). The interaction time of the $\mathbf{H F}$ and $\mathbf{L F}$ before breaking up is related to the angle $\alpha$. The coloring denotes the composition of the fragments. Blue corresponds to neutron richness and red to relative neutron deficiency. The $\mathrm{TLF}^{*}$ is in gray since it is not measured in this analysis.

on the daughters of the PLF*, with the understanding that in the symmetric collisions the same features should exist for the daughters of the TLF* [16].

Events where at least two charged fragments were measured in NIMROD were analyzed. Fragments were sorted by atomic number and charge-identical fragments were sorted by atomic mass. The fragment with the larger atomic number is referred to as the "heaviest fragment" or HF. The fragment with the second largest atomic number is referred to as the "lighter fragment" or LF. Events were selected in which $Z_{H} \leq 12$ and $Z_{L} \leq 3$. A total charge of all charged fragments detected in an event of $21 \leq \mathrm{Z}_{\text {tot }} \leq 32$ was also required to ensure most $\mathbf{H F}$ and $\mathbf{L F}$ originate from the PLF*. Isotopic identification was required for all fragments analyzed.

As evidence that the $\mathbf{H F}$ and $\mathbf{L F}$ are daughters of the PLF* in the mechanism illustrated in Figure 1, the parallel velocity distributions of the $\mathbf{H F}$ (red) and $\mathbf{L F}$ (blue) are presented in Figure 2. The velocity of the beam $(\mathrm{v}=0.27 c)$ and mid-velocity $(\mathrm{v}=0.13 c)$ are indicated by the two dashed lines. The velocity of the HF is peaked just below beam velocity consistent with velocity damping of the PLF* due to its interaction with the TLF*. The velocity distribution of the $\mathbf{L F}$ is peaked at lower velocities than the $\mathbf{H F}$, but is still significantly above mid-velocity. The shift of the distribution of the $\mathbf{L F}$ from that of $\mathbf{H F}$ toward midvelocity is consistent with the mechanism of dynamical decay illustrated in Figure 1. The three combinations of $Z_{H}$ and $Z_{L}$ shown in figure 2 are representative of all pairs of $Z_{H}$ and $Z_{L}$ examined. One more feature of dynamical decay is evident in Figure 2: as $Z_{L}$ is increased or as $Z_{H}$ is decreased, the parallel velocity distribution of the $\mathbf{L F}$ narrows and the $\mathbf{H F}$ broadens. The means of the distributions also shift toward each other. 

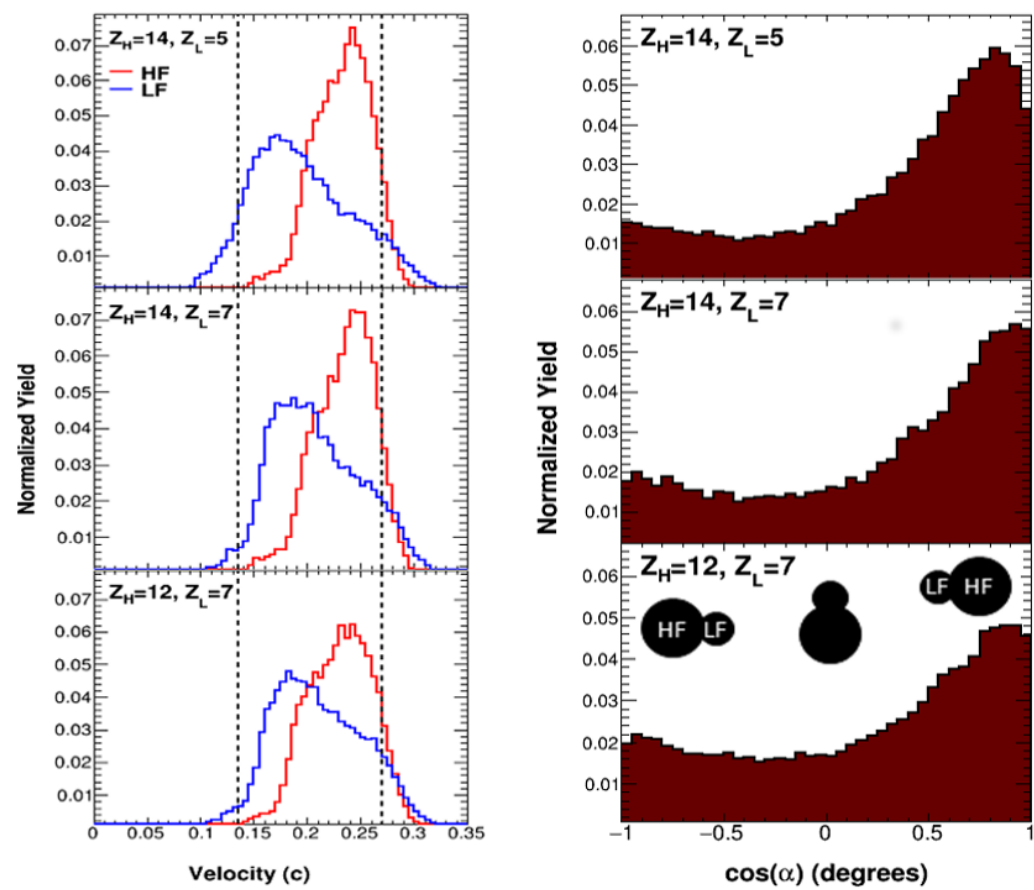

Figure 2: Velocity distribution (left) and normalized angular distributions (right) of $\mathbf{H F}$ and $\mathbf{L F}$ for selecct pairings. For the velocity distributions, the normalized yield of $\mathbf{H F}$ is the red line and $\mathbf{L F}$ is the blue line. The average velocity of both $\mathbf{H F}$ and $\mathbf{L F}$ is above mid-velocity (dotted line on left). The velocity of $\mathbf{H F}$ is centered above HF. For the angular distributions, emission of the lighter charged fragment in the backward direction (toward the target) corresponds to $\cos (\alpha)=1$.

Although only 3 pairings are shown, 43 pairings were analyzed in total. These pairings range from $Z_{H}=12-20$ and $Z_{L}=3-11$. The three pairings shown throughout this work are representative of the rest of the data set.

To focus on characteristics of dynamical decay, the alignment angular distributions are presented in Figure 2. Strong alignment is characteristic of dynamical decay. The alignment angle is defined as $\alpha=\operatorname{acos}\left(\frac{\vec{v}_{C M} \cdot \vec{v}_{R E L}}{\left\|\vec{v}_{C M}\right\| \vec{v}_{R E L} \|}\right)$. The velocity vectors in the equation are the two-fragment center-ofmass velocity $\vec{v}_{C M}=\left(m_{H F} \vec{v}_{H F}+m_{L F} \vec{v}_{L F}\right) /\left(m_{H F}+m_{L F}\right)$ and the relative velocity $\vec{v}_{R E L}=\vec{v}_{H F}-\vec{v}_{L F}$. These velocity vectors are depicted on Figure $1 \mathrm{~b}$. Since most of the nuclear collisions within the data set are peripheral and mid-peripheral collisions, the TLF* and PLF* start rotation around each other during their interaction. After the PLF* and TLF* break apart, the PLF* continues to move along the separation axis and to rotate around its center of mass (shown in Figure 1b). The PLF* will eventually break apart again at a later point in time. The rotation angle $\alpha$ is the time between the scission of the PLF*-TLF* and the scission of the PLF* into the HF and LF. $\alpha=0^{\circ}$ corresponds to $t=0$ and $\mathbf{H F}$ emitted forward relative to $\mathbf{L F}$. Therefore, larger rotation angle corresponds to a longer time during which the the $\mathbf{H F}$ and $\mathbf{L F}$ can exchange nucleons.

Figure 2 shows the angular distributions for same selected pairings. Focusing on the upper panel of Figure 2, the angular distribution is peaked at $\cos (\alpha)=1$. A noteable enhancement in the yield is seen out to $\cos (\alpha)=0.5$ followed by a flattening between $-1<\cos (\alpha)<0.5$. There is a slight 
increase in the yield close to $\cos (\alpha)=-1$. The same trend is seen in the middle and bottom panel indicating the same reaction dynamics at work.

The enhanced yield at $\cos (\alpha)=1$ is understood when considering the decay mechanisms. The first decay mechanism is standard statistical decay, which is present at all angles of rotation. For a non-rotating PLF* before decay, the $\cos (\alpha)$ distribution should be flat. As the angular momentum increases, the yield at $\cos (\alpha)= \pm 1$ increases since fragments are likely to decay in its rotational plane similar to a wet dog shaking off water. The angular distribution retains its symmetry around $\cos (\alpha)=0$. The composition of angular distribution between $-1<\cos (\alpha)<0$ is mostly statistical decay.

The large yield present at $\cos (\alpha)>0$ is due mostly to dynamical decay, which breaks up along the PLF*-TLF* separation axis. Since this break up occurs within a very short timescale, dynamical decay has the largest yield close to $\cos (\alpha)=1$ with a sharp decrease as the angle of rotation increases. There is almost no contribution past $\cos (\alpha)=0$. The largest peak corresponding to dynamical decay is seen for the pairing with the greatest charge difference $\left(Z_{H}=14, Z_{L}=7\right)$. As the charge difference decreases (moving from the top panel to the bottom panel), the dynamical decay peak decreases and the distribution broadens. This trend is in agreement with previous trends [17]. Due to large collectivity and a large potential barrier, charge symmetric splits are slowed and their likelihood is decreased. This behavior supports dynamical production of $\mathbf{H F}$ and $\mathbf{L F}$ seen in Figure 2. The large peak is also indicative of a short decay timescale relative to the rotational period of the PLF*, which is the motivation behind using the angle of rotation as a surrogate for for time.

To make sure the features seen in the angular distributions are not created by the detector array, the angular distributions were replicated using the statistical model GEMINI++ [25] and were filtered through a NIMROD software filter. The symmetry of the statistical decay distribution was preserved indicating that the asymmetry of the angular distribution, which is attributed to dynamical decay, was not artifically produced by the detector array.

The asymmetry, $\langle\Delta\rangle=\left\langle\frac{N-Z}{A}\right\rangle$, was calculated as a function of the rotational angle $(\alpha)$ for all $Z_{H}, Z_{L}$ pairings. Results are shown in Figure 3 for the same pairings seen in Figure 2. The high statistics and near complete angular range allows for detailed characterization of the evolution of the NZ equilibration. The $\left\langle\Delta_{L}\right\rangle$ starts off neutron-rich at $\alpha=0^{\circ}$ or $t=0$. As the angle of rotation increases, the composition decreases rapidly for small angles of rotation followed by leveling off at $\alpha \approx 80^{\circ}$. Similarly, the $\left\langle\Delta_{H}\right\rangle$ starts off relatively neutron-poor and evolves to be more neutron-rich as the angle of rotation increases. $\left\langle\Delta_{H}\right\rangle$ also plateaus at $\alpha \approx 80^{\circ}$. The evolution of the composition is exponential in nature; this is consistent with first-order kinetics. The inset in Figure 3 depicts the change in NZ composition as a function of corresponding angle. Blue represents neutron abundance and red represents relative neutron deficiency.

The evolution of the composition can be understood by considering the dynamical mechanism. Dynamically produced fragments are highly deformed. Due to this deformation and a velocity gradient, the PLF* breaks apart quickly along the PLF*-TLF* separation axis (which is also the deformation axis). Material from the neck region is relatively neutron-rich due to preferential flow of neutrons to minimize the symmetry energy [26]. If the fragments break apart immediately, the neutron-rich composition of the neck (now $\mathbf{L F}$ ) is maintained. The relatively neutron-poor composition of the PLF (now HF) is also preserved. At the same time, attractive forces are at work delaying $\mathbf{H F}$ and $\mathbf{L F}$ decay. Surface tension also drives the PLF* towards sphericity. As long as 


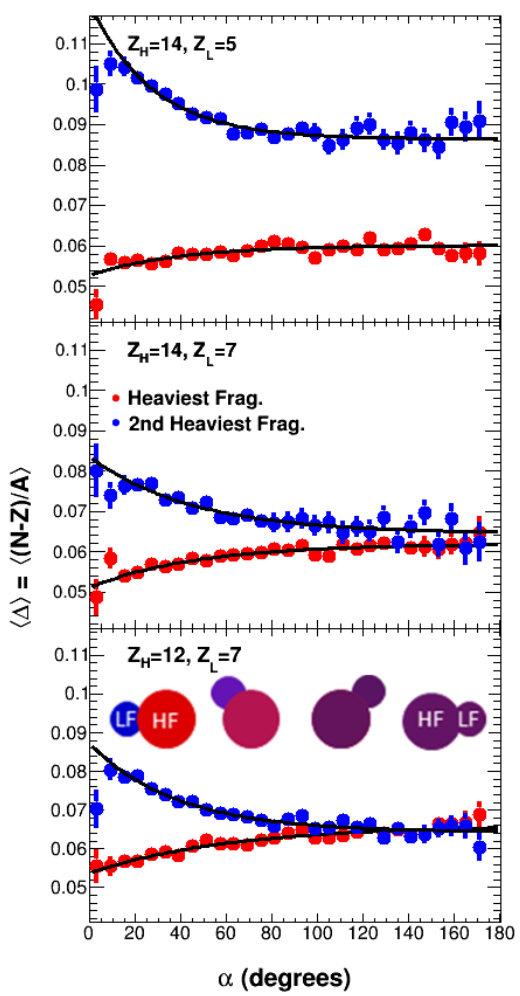

Figure 3: Composition as a function of angle of rotation showing equilibration for the same $Z_{H}$ and $Z_{L}$ pairings shown in Fig. 4. As the angle of rotation increases $\left(\alpha>0^{\circ}\right)$, the $\left\langle\frac{N-Z}{A}\right\rangle$ decreases for $Z_{L}$ and increases for $Z_{H}$ before plateauing. This indicates the majority of equilibration occurs before $\approx 80^{\circ}$.

the fragments are in contact, they can exchange nucleons.

However, the asymptotic values of $\Delta_{H}$ and $\Delta_{L}$ are not necessarily the same. Thermodynamics suggest the final chemical potentials, not asymptotic compositions, should be the same. The chemical potential depends on the internal energy, density and ground-state binding energies. Due to mass conservation, the change in the $\left\langle\Delta_{H}\right\rangle$ is smaller than the change in $\left\langle\Delta_{L}\right\rangle$.

In order to characterize the equilibration kinetics, $\left\langle\Delta_{H}\right\rangle$ and $\left\langle\Delta_{L}\right\rangle$ were fit with an exponential of the form $\langle\Delta\rangle=a+b \cdot \exp (-c \alpha)$. The exponential fits are shown as the black lines in Figure 3. The data is well described by the exponential fits providing evidence that NZ equilibration follows first order kinetics. The $c$ value is the rate constant in inverse degrees and can be converted to time as discussed in the next paragraph. The average rate constant was $0.02 \pm 0.01$ per degree for $\mathbf{H F}$ and $0.03 \pm 0.01$ per degree for $\mathbf{L F}$. These values are consistent within experimental uncertainty indicating that the force driving the equilibration is only dependent on the difference in $\mathbf{H F}$ and $\mathbf{L F}$ chemical composition. The driving force is independent of size of the reaction partners.

The rate constant can be converted from degrees to time if the correlation between the rotation angle and time is monotonic. The large yield at $\cos (\alpha)=1$ in Figure 2 suggests that the decay time is much shorter than the rotational period. If this were not the case, the distribution would appear symmetric around $\cos (\alpha)=0$. The equation used to convert the angle of rotation to time is $t=\alpha / \omega$, 
where $t$ is the time and $\omega$ is the angular frequency. This assumes the relationship between the angle of rotation and time is linear. The angular frequency is $\omega=(J \hbar) / I_{e f f}$, where $J$ is the angular momentum and $I_{\text {eff }}$ is the moment of inertia. The moment of inertia is calculated assuming two touching spheres, $\mathbf{H F}$ and $\mathbf{L F}$, rotating around their common center of mass $\left(I_{\text {eff }}=m_{H} r_{C M, H}^{2}+\right.$ $\left.\frac{2}{5} m_{H} r_{H}^{2}+m_{L} r_{C M, L}^{2}+\frac{2}{5} m_{L} r_{L}^{2}\right) \cdot r_{H}$ is the radius of $\mathbf{H F}, r_{L}$ is the radius of $\mathbf{L F}, r_{C M, H}$ is the distance from the center of $\mathbf{H F}$ to their center-of-mass, and $r_{C M, L}$ is the distance from the center of $\mathbf{L F}$ to their center-of-mass. $m_{H}$ and $m_{L}$ were the masses of $\mathbf{H F}$ and $\mathbf{L F}$ respectively. The moment of inertia was calculated to be between $2.8-9.9 \times 10^{-42} \mathrm{MeV} \cdot \mathrm{s}^{2}$.

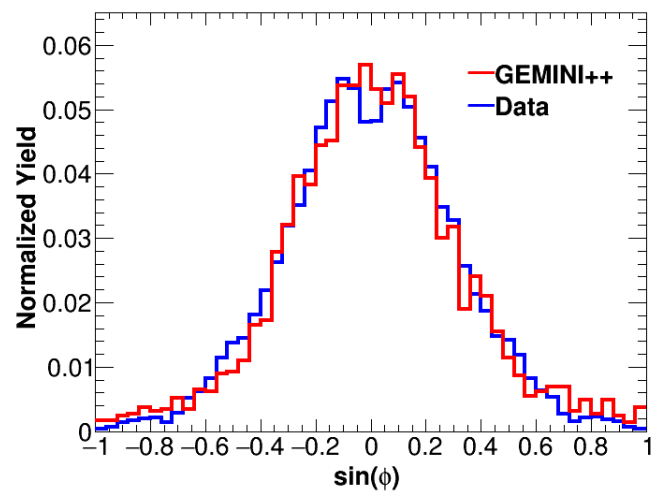

Figure 4: Out-of-plane $\alpha$-particle distribution for the experimental data (blue) and the results of the statistical model GEMINI++ (red). An angular momentum of $22 \hbar$ was the input value for GEMINI++.

The angular momentum was calculated using the width of the out-of-plane $\alpha$-particle distributions from fission studies [27]. These studies have determined the angular momentum of the combined species is proportional to the width of the out-of-plane fragment distribution. The spin axis is perpendicular to the reaction plane, which is defined by the beam axis and the $\mathbf{H F}$ and $\mathbf{L F}$ center-of-mass velocity vector $\vec{v}_{C M}$. The angle relative to the reaction plane, $\phi$, was calculated for all coincident $\alpha$-particles. The experimental distribution is represented by the blue line in Figure 4 . The experimental data was fit with a gaussian and a width of 0.28 was extracted. The variation is very minimal for different pairings of $Z_{H}, Z_{L}$. The distributions were reproduced with GEMINI++ varying the angular momentum and excitation energies. The value ranged from a spin of $10 \hbar$ and excitation energy of $\mathrm{E}^{*} / \mathrm{A}=0.8 \mathrm{MeV}$ ) to a spin of $50 \hbar$ and an excitation energy of $\mathrm{E}^{*} / \mathrm{A}=1.2 \mathrm{MeV}$. Given the difficulty in pinpointing the exact spin and excitation energy, the geometric mean of $22 \hbar$ was used. This produced a systematic uncertainty of a factor of 2.2. The GEMINI++ results correspond to the red line in Figure 4. The rotational periods extracted are between 1 and $4 \mathrm{zs.} \mathrm{The}$ average is $3 \pm_{1}^{6}$ zs. The results for the pairings shown and the average of all are seen in Table 1 .

The NZ composition of deformed, out-of-equilibrium nuclei was examined as a function of time. The composition of the two heaviest fragments originating from the PLF* is observed to evolve exponentially over time. The timescale was extracted using the angle of rotation as a surrogate and taking advantage of the short decay time relative to the rotational period. The timedependent measurement of the NZ equilibration can be used to further constrain the nuclear equation of state. The measurement presented in this proceeding is the first measurement showing NZ 


\begin{tabular}{|c|c|c|c|c|c|}
\hline$Z_{H}, Z_{L}$ Pairing & Period $(\mathrm{zs})$ & $k_{H}\left(\mathrm{zs}^{-1}\right)$ & $k_{L}\left(\mathrm{zs}^{-1}\right)$ & $\tau_{H}(\mathrm{zs})$ & $\tau_{L}(\mathrm{zs})$ \\
\hline 12,7 & $3 \pm_{1}^{6}$ & $2 \pm_{1}^{3}$ & $4 \pm_{2}^{4}$ & $0.5 \pm_{0.2}^{1}$ & $0.3 \pm_{0.1}^{0.6}$ \\
âĂ ̌ 14,5 & $2 \pm_{1}^{5}$ & $4 \pm_{3}^{5}$ & $6 \pm_{3}^{7}$ & $0.3 \pm_{0.1}^{0.6}$ & $0.2 \pm_{0.1}^{0.4}$ \\
14,7 & $3 \pm_{1}^{7}$ & $3 \pm_{2}^{3}$ & $3 \pm_{2}^{3}$ & $0.4 \pm_{0.2}^{0.9}$ & $0.4 \pm_{0.2}^{0.8}$ \\
Average & $3 \pm_{1}^{6}$ & $3 \pm_{2}^{4}$ & $4 \pm_{2}^{4}$ & $0.3 \pm_{0.2}^{0.7}$ & $0.3 \pm_{0.1}^{0.6}$ \\
\hline
\end{tabular}

Table 1: Rotational period, rate constants for $\mathbf{H F}\left(\mathrm{k}_{H}\right)$ and $\mathbf{L F}\left(\mathrm{k}_{L}\right)$, and the mean lifetime for $\mathbf{H F}\left(\tau_{H}\right)$ and $\mathbf{L F}\left(\tau_{L}\right)$. The $\left(Z_{H}, Z_{L}\right)$ pairings chosen in the table are the same as shown throughout the analysis. The last value is the average of all 43 pairings.

equilibration follows first-order kinetics. The rate constant extracted was approximately $3 \mathrm{zs}^{-1}$, corresponding to a mean lifetime of equilibration of $0.3 \mathrm{zs}$.

In 2004, Tsang et al. [11] used BUU simulations to demonstrate that NZ equilibration is sensitive to the form of the nuclear equation of state employed in the model. The simulation showed the composition is dependent on the contact time of the fragments, the driving potential and the initial asymmetry of the target and projectile. Much research has focused on the asymptotic composition values. Measuring the composition as a function of time can give more insight into the nuclear equation of state. Through applying this technique to data, theoretical models as well as the understanding of fundamental interactions of nuclear materials can be enhanced.

\section{Acknowledgements}

This work was made possible by support from the DOE (DE-FG02-93ER40773) and the Robert A. Welch Foundation (A-1266). We thank the staff of the TAMU Cyclotron Institute for the excellent particle beams and technical support. We gratefully acknowledge Kyle Brown, Sylvie Hudan, Joe Natowitz and Lee Sobotka for critical reading of the manuscript. We would further like to thank Robert Charity for use of GEMINI++ code.

\section{References}

[1] U. Garg et al. The giant monopole resonance in the sn isotopes: Why is tin so fluffy? Nuc. Phys. A, 788:36c, 2007.

[2] V. Baran et al. Reaction dynamics with exotic nuclei. Phys. Rept., 410:335, 2005.

[3] X. Roca-Maza et al. Nuclear skin of Pb-208, nuclear symmetry energy, and the parity radius experiment. Phys. Rev. Lett., 106:252501, 2011.

[4] P.B. Demorest et al. A two-solar-mass neutron star measured using Shapiro delay. Nature, 467:1081, 2010.

[5] J.M. Lattimer and M. Prakash. The physics of neutron stars. Science, 304:536-542, 2004.

[6] M. A. Famiano et al. Neutron and proton transverse emission ratio measurements and the density dependence of the asymmetry term of the nuclear equation of state. Phys. Rev. Lett., 97:052701, 2006. 
[7] M.B. Tsang et al. Constraints on the density dependence of the symmetry energy. Phys. Rev. Lett., 102:122701, 2009.

[8] M.B. Tsang et al. Isoscaling in statistical models. Phys. Rev. C, 64:054615, 2001.

[9] S. Galanopoulos et al. Isoscaling of mass reconstructed quasiprojectiles from collisions in the fermi energy regime. Nuc. Phys. A, 837:145, 2010.

[10] G.A. Souliotis et al. Isoscaling of heavy projectile residues and N/Z equilibration in peripheral heavy-ion collisions below the Fermi energy. Phys. Rev. C, 90:064612, 2014.

[11] M.B Tsang et al. Isospin diffusion and the nuclear symmetry energy in heavy ion reactions. Phys. Rev. Lett., 92:062701, 2004.

[12] M.B Tsang et al. Constraints on the symmetry energy and neutron skins from experiments and theory. Phys. Rev. C, 86:015803, 2012.

[13] L. May. Isospin Equilibration in Fermi-Energy Heavy-Ion Nuclear Collisions. PhD thesis, Texas A\&M University, 2015.

[14] F. Bocage et al. Dynamical effects in nuclear collisions in the Fermi energy range: aligned breakup of heavy projectiles. Nucl. Phys. A, 676:391, 2000.

[15] B. Davin et al. Fragment production in noncentral collisions of intermediate-energy heavy ions. Phys. Rev. C, 65:064614, 2002.

[16] J. Colin et al. Dynamical effects in multifragmentation at intermediate energies. Phys. Rev. C, 67:064603, 2003.

[17] A.B. McIntosh et al. Short-lived binary splits of an excited projectile-like fragment induced by transient deformation. Phys. Rev. C, 81:034603, 2010.

[18] J. Galin et al. The transition between quasi elastic and completely damped collisions studied in the ${ }^{40} \mathrm{Ar}(280 \mathrm{MeV})+{ }^{58} \mathrm{Ni}$ reaction. Z. Physik A, 278:347-352, 1976.

[19] L.G. Moretto and R.P. Schmitt. Deep inelastic reactions: a probe of the collective properties of nuclear matter. Rep. Prog. Phys., 44:533, 1981.

[20] H. Freisleben and J.V. Kratz. N/Z-equilibration and nucleon exchange in dissipative heavy-ion collisions. Phys. Rep., 106:1, 1984.

[21] S. Hudan et al. Tracking saddle-to-scission dynamics using N/Z in projectile breakup reactions. Phys. Rev. C, 86:021603, 2012.

[22] K. Brown et al. Timescale for equilibration of N/Z gradients in dinuclear systems. Phys. Rev. C, 87:061601, 2013.

[23] S. Hudan and R.T. deSouza. Timescale for isospin equilbration in projectile breakup. Eur. Phys. J. A, 50:36, 2014.

[24] S. Wuenschel et al. NIMROD-ISiS, a versatile tool for studying the isotopic degree of freedom in heavy ion collisions. NIMA, 604:578, 2009.

[25] R.J. Charity. N-Z distributions of secondary fragments and the evaporation attractor line. Phys. Rev. C, 58:1073, 1998.

[26] D. Theriault et al. Neutron-to-proton ratios of quasiprojectile and midrapidity emission in the ${ }^{64} \mathrm{Zn}+{ }^{64} \mathrm{Zn}$ reaction at $45 \mathrm{MeV} /$ nucleon. Phys. Rev. C, 74:051602, 2006.

[27] R. Vandenbosch and J.R. Huizenga. Nuclear Fission. Academic Press, New York, 1973. 